\title{
Prevalência de tuberculose no Estado do Pará entre 2015-2019
}

\section{Prevalence of tuberculosis in the State of Pará between 2015-2019}

Prevalencia de tuberculosis en el Estado de Pará entre 2015-2019

Tanise Nazaré Maia Costa ORCID: https://orcid.org/0000-0003-1319-5591 Centro Universitário do Estado do Pará, Brasil

E-mail: tanise.costa@prof.cesupa.br

Rhomero Salvyo Assef Souza

ORCID: https://orcid.org/0000-0002-2109-9674 Centro Universitário do Estado do Pará, Brasil

E-mail: rhomeroassef@yahoo.com.br

Roberto José Falcón Tamargo

ORCID: https://orcid.org/0000-0001-7852-3475 Centro Universitário do Estado do Pará, Brasil E-mail: rftamargo@yahoo.es

Elaine Vilhena de Freitas ORCID: https://orcid.org/0000-0003-3987-6555 Centro Universitário do Estado do Pará, Brasil E-mail: elainefreitasv@gmail.com

Laura Correa de Souza

ORCID: https://orcid.org/0000-0002-2315-610X

Centro Universitário do Estado do Pará, Brasil

E-mail: lauracorrea_souza@hotmail.com

Yasmin Batista Mendes

ORCID: https://orcid.org/0000-0003-3713-0936

Centro Universitário do Estado do Pará, Brasil

E-mail: ybmmendes@gmail.com

Bárbara Vitória Lobato Roriz

ORCID: https://orcid.org/0000-0001-5542-0748

Centro Universitário do Estado do Pará, Brasil

E-mail: barbaravitoriar@gmail.com

Virginia Márcia Peixoto Montes

ORCID: https://orcid.org/0000-0003-2434-8143

Centro Universitário do Estado do Pará, Brasil

E-mail: virginiampeixoto68@gmail.com

Isabela Martins Viana

ORCID: https://orcid.org/0000-0002-3422-856X

Centro Universitário do Estado do Pará, Brasil

E-mail: isabela.martins.viana@gmail.com

Alessandra Belo da Costa

ORCID: https://orcid.org/0000-0001-5105-6766

Centro Universitário do Estado do Pará, Brasil

E-mail: alessandra19250034@aluno.cesupa.br

Hemannuella da Silva Costa

ORCID: https://orcid.org/0000-0003-3159-0990

Centro Universitário do Estado do Pará, Brasil

E-mail: hemannuellacosta@gmail.com

Raphael Kerber Almeida

ORCID: https://orcid.org/0000-0003-1871-9294

Centro Universitário do Estado do Pará, Brasil

E-mail: rapha.kerber@gmail.com

\begin{abstract}
Resumo
Objetivou-se descrever sobre a prevalência de tuberculose em alguns municípios do estado do Pará entre os anos de 2015 a 2019 e colher informações sobre a doença. A pesquisa é de caráter descritivo, observacional e retrospectivo por meio de levantamento de dados obtidos pelo site do Ministério da Saúde e pelo banco de dados de tuberculose no DATASUS para analisar epidemiológica e numericamente os casos de tuberculose registrados nestes municípios, registrados entre os anos de 2015 a 2019. Com os dados encontrados observou-se que houve estabilidade durante os últimos 5 anos, no entanto, ocorreu um aumento substancial do ano de 2018 para 2019 no município de Marituba. Com relação a faixa etária, no período de cinco anos de pessoas com tuberculose foi entre 20-39 anos e 40-59 anos
\end{abstract}


com maior número de casos no munícipio de Belém. Denotou-se, ainda, que os homens lideraram a pesquisa com predominância dos casos no município de Belém. Conclui-se, que mediante esta pesquisa, será factível capacitar os profissionais de saúde para possíveis casos de surto ou de epidemias futuras, estimar quantidades mais precisas de medicamentos, demanda de recursos sobre a manutenção da infraestrutura, entre outras medidas de intervenção.

Palavras-chave: Tuberculose; Prevalência; Atenção à saúde.

\begin{abstract}
The goals was to relate the prevalence of tuberculosis in some cities in the state of Pará between the years 2015 to 2019 and accumulated details about the disease. The research is descriptive, observational and retrospective through a view of data obtained from the Ministry of Health website and the tuberculosis database at DATASUS to analyze epidemiologically and numerically the cases of tuberculosis registered in the municipalities, registered between the years 2015 to 2019 . With the data found, it was perceived that there was stability during the last 5 years, however, there was a substantial increase from 2018 to 2019 in the city of Marituba. Regarding the age group, in the five-year period of people with tuberculosis, it was between 20-39 years old and 40-59 years old, with the highest number of cases in the municipality of Belém. of cases in the municipality of Belém. It is concluded that through this research, it will be feasible to train health professionals for possible cases of outbreaks or future epidemics, estimate more precise amounts of medicines, demand for resources on the maintenance of infrastructure, among other intervention measures.

Keywords: Tuberculosis; Prevalence; Health care.

\section{Resumen}

El objetivo fue describir la prevalencia de tuberculosis en las ciudades del estado de Pará entre los años 2015 a 2019 y recopilar información sobre la enfermedad. La investigación es descriptiva, observacional y retrospectiva a través de una encuesta de datos obtenidos del sitio web del Ministerio de Salud y la base de datos de tuberculosis en DATASUS para analizar epidemiológica y numéricamente los casos de tuberculosis registrados en los municipios, registrados entre los años 2015 a 2019. Con los datos encontrados, se observó que hubo estabilidad durante los últimos 5 años, sin embargo, hubo un incremento sustancial de 2018 a 2019 en la ciudad de Marituba. En cuanto al grupo de edad, en el quinquenio de personas con tuberculosis, fue entre 20-39 años y 40-59 años con el mayor número de casos en el municipio de Belém. De casos en el municipio de Belém. Se concluye que a través de esta investigación será factible capacitar a los profesionales de la salud para posibles casos de brotes o futuras epidemias, estimar cantidades más precisas de medicamentos, demanda de recursos para el mantenimiento de infraestructura, entre otras medidas de intervención.
\end{abstract}

Palabras clave: Tuberculosis; Prevalencia; Atención a la salud.

\title{
1. Introdução
}

A tuberculose (TB) é uma doença infecciosa causada pelo bacilo de Mycobacterium tuberculosis considerada uma epidemia global segundo a Organização Mundial da Saúde (OMS) (Cortez et al, 2021; World Health Organization, 2017). A doença acomete principalmente os pulmões e pode ocasionar o óbito caso não seja adequadamente tratada. Adicionalmente, é uma enfermidade propagada de forma facilitada por via aérea de uma pessoa contaminada, disseminando bacilos no ambiente, a outro indivíduo, ao exalar de aerossóis oriundos da tosse, fala ou espirro (Silva et al, 2020; Silva et al, 2018).

Calcula-se que 10,4 milhões de pessoas ficaram doentes devido a TB em 2016, sendo que foram notificados 6,3 milhões de novos casos de tuberculose. Em 2017, o Ministério da Saúde divulgou um planejamento para eliminar a TB - o Plano Nacional pelo Fim da Tuberculose como Problema de Saúde Pública - almejando a redução, até 2035, da incidência de tuberculose para menos de 10 casos/100.000 habitantes e a mortalidade por tuberculose menor que 1 morte/100.000 habitantes (Silva et al, 2018). Em 2019, no Brasil foram notificados 73.864 mil casos da doença representando número expressivo (Agência Brasil, 2020).

Essa infecção, anteriormente chamada como tísica, foi descoberta, no século XIX, como peste branca, ceifando milhares de vidas em todo o mundo. Em torno da metade do século XX, uma diminuição abrupta de novos casos e da mortalidade relacionadas à $\mathrm{TB}$, foi denotada em países desenvolvidos, principalmente com a melhoria das condições de vida das populações (Massabni \& Bonini, 2019; Guimarães et al, 2018; Menezes \& Moura, 2021).

Em meados da década de 1980, ocorreu agravamento global da TB. Nos países denominados "ricos", esse recrudescimento existiu, sobretudo, à emergência da infecção pelo Vírus da Imunodeficiência Humana (HIV) e, nos países de 
baixa renda, devido à ampliação da miséria e do processo de urbanização descontrolada, além de desestruturação dos serviços de saúde e dos programas de controle da tuberculose (Alcade et al, 2018; Bastos et al, 2019).

A TB é uma patologia totalmente prevenível e curada, porém persiste em situações de pobreza e auxilia na continuação da desigualdade social, incluindo brasileiros privados de liberdade e indígenas (Oliveira, Ribeiro \& Dellanhese, 2020; Moreira et al, 2019, Hino et al, 2021, Ferreira et al, 2020).

Em 2018, em torno de 30\% dos casos de TB no Brasil foram diagnosticados durante internação hospitalar por piora de quadro clínico, gerando ônus exorbitante ao Sistema Único de Saúde (SUS) comparados com tratamento e prevenção na Atenção Básica (Almeida et al, 2020.

Durante o ano de 2020, a vivência na pandemia de COVID-19 no Brasil, tornou necessária a busca por informações sobre outras doenças respiratórias acometidas (Guerra et al, 2021; Maciel \& Silva, 2021). Outrossim, observou-se a suspeita da doença sobre alguns pacientes, o que despertou curiosidade e discussão entre os especialistas.

Diante desse quadro alarmante, observou-se a necessidade de descrever a prevalência de tuberculose em alguns municípios do Estado do Pará entre os anos de 2015 a 2019. Logo, foi estabelecido o interesse por uma intervenção social a qual pudesse envolver tanto profissionais da saúde quanto moradores das comunidades. Por isso, pesquisar sobre a situação da doença nos períodos mais recentes (2015 a 2019), é o primeiro passo para atenuar o coeficiente de infecção, uma vez que há poucos investimentos na área da saúde (Sousa et al., 2020).

\section{Metodologia}

Este trabalho é de caráter descritivo, observacional e retrospectivo por meio de dados obtidos pelo site do Ministério da Saúde e pelo banco de dados de tuberculose no DATASUS para analisar numérica e epidemiologicamente os casos de tuberculose registrados em alguns municípios do Pará, registrados entre os anos de 2015 a 2019.

Para a presente pesquisa foram realizados levantamentos sobre o assunto, considerando sexo, faixa etária, escolaridade e raça mais prevalente de infectados nessa região; e assim identificar em quais subgrupos há maior propensão de adquiri-la. Os dados foram catalogados no Excel e expostos graficamente.

Não houve necessidade do uso do Termo de Consentimento Livre e Esclarecido (TCLE) e da submissão à Comissão de Ensino e Pesquisa (CEP), por se tratar de um estudo descritivo de dados já existentes em bancos de dados públicos. Além disso, não foi imprescindível o Termo de Consentimento de Uso de Dados (TCUD) e autorização do responsável/coordenador da unidade da ESF, por se tratar apenas de uma revisão de dados do Ministério da Saúde e SINAN.

\section{Resultados e Discussão}

As bactérias que causam a tuberculose são espalhadas quando uma pessoa infectada tosse ou espirra o que facilita a propagação da doença. Vista a partir de um quadro nacional, a TB apresenta muitos casos no Brasil (Silva et al, 2019). Dessa forma, foi feita uma análise da infecção respiratória em alguns municípios que fazem parte do Estado do Pará, com foco naqueles que apresentaram um maior número de casos, são eles: Belém, Ananindeua e Marituba.

Em análise, ao número total de casos em alguns municípios paraenses, segundo dados do DATASUS (Tabela 1), observa-se nos últimos cinco anos a predominância dos casos na capital Belém, município o qual apresentou o maior número de infectados de 2015-2019, apresentando uma constância nos dados todos os anos, com leves aumentos e quedas, a exemplo do ano de 2015-1737-casos e no ano de 2019-1884 casos, o município obteve um aumento de 8\% no número de casos, comparando o primeiro e o último ano.

Em seguida, o segundo município que apresentou o maior número de casos foi o município de Ananindeua que também mostrou dados constantes, porém sofreu um aumento de $60 \%$ no número de casos entre o início e o final da pesquisa, 
com 323 casos em 2015 e 518 casos em 2019. Foi observado também um aumento significativo no número de casos na região de Marituba com aumento de 195\% no número de casos em comparação com o início da pesquisa, com 127 casos em 2015 e 375 casos em 2019. Assim, percebe-se um número permanente de casos na região de Belém e um aumento de casos na região de Ananindeua e Marituba.

Esse dado de aumento em Marituba de um ano para outro apenas de forma pontual pode ser explicado, provavelmente, por um somatório de notificações que estavam atrasadas para serem colocadas em internet ou acrescidas por subnotificações.

Casos de subnotificação são frequentes, como se observou no estudo de Santos et al (2018), em Recife, com proporção de $29 \%$ de subnotificação de TB, e os fatores associados foram: apresentar forma clínica da TB pulmonar cavitária ou não especificada, ou ter os dois tipos de TB ao mesmo tempo; ou ser atendido fora da cidade onde fora realizada a pesquisa.

Tabela 1: Casos confirmados de tuberculose em alguns municípios do Estado do Pará.

\begin{tabular}{|l|c|c|c|c|c|}
\hline & Ananindeua & Belém & Benevides & Marituba & Santa Bárbara do Pará \\
\hline $\mathbf{2 0 1 5}$ & 323 & 1737 & 36 & 127 & 6 \\
\hline $\mathbf{2 0 1 6}$ & 329 & 1732 & 38 & 136 & 18 \\
\hline $\mathbf{2 0 1 7}$ & 340 & 1820 & 29 & 140 & 19 \\
\hline $\mathbf{2 0 1 8}$ & 409 & 1726 & 52 & 189 & 20 \\
\hline $\mathbf{2 0 1 9}$ & 518 & 1884 & 47 & 375 & 31 \\
\hline
\end{tabular}

Fonte: DATASUS.

Ao pesquisarmos os casos de tuberculose e separarmos por sexo, os homens lideram a pesquisa com predominância dos casos no município de Belém, de acordo com os dados obtidos, a população masculina atingida pela doença é o dobro da feminina como mostra os dados de 2018 em que o número de homens foi de 1112 casos e o número de mulheres 614 casos na capital paraense (Tabelas 1 e 2).

Além disso, com a primazia da população parda como a mais atingida. Assim, verifica-se que a população masculina parda é a mais infectada pela doença.

Os achados concordaram com o estudo de Silva et al (2020), realizados em um município nordestino, o qual encontrou a tuberculose acometendo mais homens $(63,2 \%)$ e pardos $(60,2 \%)$.

Em um trabalho realizado em idosos com TB por Ferreira et al (2021), foi encontrado uma diferença entre os gêneros em relação a forma de acometimento, no qual o sexo feminino é mais assolado por TB extrapulmonar (14\%) e o masculino, à forma pulmonar (89\%).

Tabela 2: Casos confirmados de tuberculose entre homens em alguns municípios do Estado do Pará.

\begin{tabular}{|l|c|c|c|c|c|}
\hline & Ananindeua & Belém & Benevides & Marituba & Santa Bárbara do Pará \\
\hline $\mathbf{2 0 1 5}$ & 196 & 1086 & 20 & 90 & 3 \\
\hline $\mathbf{2 0 1 6}$ & 203 & 1079 & 27 & 90 & 11 \\
\hline $\mathbf{2 0 1 7}$ & 197 & 1168 & 22 & 113 & 12 \\
\hline $\mathbf{2 0 1 8}$ & 277 & 1112 & 5 & 157 & 12 \\
\hline $\mathbf{2 0 1 9}$ & 313 & 1190 & 24 & 331 & 15 \\
\hline
\end{tabular}

Fonte: DATASUS. 
Tabela 3: Casos confirmados de tuberculose entre mulheres em alguns municípios do Estado do Pará.

\begin{tabular}{|l|c|c|c|c|c|}
\hline & Ananindeua & Belém & Benevides & Marituba & Santa Bárbara do Pará \\
\hline $\mathbf{2 0 1 5}$ & 127 & 651 & 16 & 37 & 3 \\
\hline $\mathbf{2 0 1 6}$ & 126 & 653 & 11 & 46 & 7 \\
\hline $\mathbf{2 0 1 7}$ & 143 & 652 & 7 & 27 & 7 \\
\hline $\mathbf{2 0 1 8}$ & 132 & 614 & 18 & 32 & 8 \\
\hline $\mathbf{2 0 1 9}$ & 205 & 694 & 23 & 44 & 16 \\
\hline
\end{tabular}

Fonte: DATASUS.

Em sequência e adicionalmente, através dos dados obtidos a partir do Ministério da Saúde, a faixa etária prevalente no período de cinco anos de pessoas infectadas pela tuberculose foi da população entre 20-39 anos e 40-59 anos com maior número de casos no munícipio de Belém, a exemplo de 2017 em que os casos de 20-39 anos foram de 794 infectados e de 4059 anos foram de 577 infectados. É valido ressaltar também a região de Marituba que apresentou um grande aumento no número de casos entre a faixa etária de 20-39 anos entre os anos de 2017 a 2019, na qual se observou um aumento de 170\% no número de casos. Desse modo, torna-se evidente que a população jovem é a mais atingida pela doença. Dados semelhantes na pesquisa de Lima et al (2019) foram encontrados, revelando que $68,11 \%$ dos portadores da doença eram homens, na faixa etária de 20-49 anos $(45,12 \%)$.

Inclui-se ainda, os dados acerca da escolaridade da população portadora de TB em algumas cidades paraenses, os quais durante o período da pesquisa foram bastante negligenciados. Tendo em vista que, somente no ano de 2019, em 25\% das notificações, pelo DATASUS, a informação escolaridade foi preenchida em branco. No entanto, entre os dados obtidos o nível escolar que mais prevaleceu entre os portadores de tuberculose foi de $5^{\circ}$ a $8^{\circ}$ série incompleta do ensino fundamental e ensino médio completo, com predominância na capital Belém, a exemplo do ano $20195^{\circ}-8^{\circ}$ série incompleta do EF -334 casos e ensino médio completo-344 casos.

Contudo, é valido relatar alguns aumentos significantes que ocorreram no decorrer dos cinco anos analisados, como em Marituba onde o número de casos entre a população de $5^{\circ}-8^{\circ}$ série incompleta do $\mathrm{EF}$ aumentou 221,7\% comparando o primeiro e o último ano da pesquisa e também em Ananindeua onde o número de casos de pessoas com ensino médio completo aumentou $100 \%$.

Desse modo, é possível afirmar que a tuberculose atinge majoritariamente a população com baixo nível de escolaridade e também a população concluinte do ensino médio, fato que gera uma contradição visto que pessoas com um maior grau de cognição deveriam ser mais informadas e colaboradoras aos métodos de prevenção.

Portanto, com toda análise feita pelo grupo, sobre a prevalência da tuberculose em alguns municípios do Pará. Entende-se que a tuberculose durante cinco anos de pesquisa apresentou um maior número de casos concentrados na capital Belém e apresentou aumentos consideráveis nas regiões de Ananindeua e Marituba, sendo adquirida principalmente por uma parcela jovem da população, em sua maioria homens de cor parda, predominantemente de baixo e médio nível escolar.

\section{Conclusão}

Destarte, com toda análise feita pelo grupo acerca da prevalência da Tuberculose no Estado do Pará, conclui-se que essa enfermidade, durante os últimos 5 anos, teve reduzida modificação dos números, com atenção especial no período entre 2018 e 2019 no município de Marituba. Além disso, entre os dados selecionados, identifica-se que o perfil epidemiológico mais suscetível e a população de mais risco são os homens, na idade adulta entre 20 e 59 anos, pardo e que não alcançou o ensino superior. Portanto, é importante salientar o quão necessário seria uma maior interação dos profissionais da saúde com a 
comunidade, na medida em que deveriam ocorrer palestras e orientações coletivas, com objetivo de orientar os pacientes sobre a doença, além de elaborar medidas de conscientização sobre a prevenção e tratamento, tendo em vista que esse acometimento merece atenção redobrada, pois além de diversas consequências deletérias, pode levar ao óbito.

Desse modo, mediante esta pesquisa, recomenda-se informar os profissionais de Saúde como os números dessa infecção tem impacto na qualidade de vida no Estado do Pará e a importância do seu combate para a melhora dos quadros de saúde da população local. Ademais, tal constatação torna o profissional mais capacitado a atender possíveis casos de surto ou de epidemias futuras, com os dados desta pesquisa será possível estimar quantidades mais precisas de antibióticos, demanda de plantonistas e de recursos sobre a manutenção da infraestrutura da atenção básica, entre outras medidas de intervenção.

\section{Referências}

Alcade, G. F. G., Santos, J. G. M. dos, Berro, E. C., Simioni, P. U., \& Ugrinovich L. A. (2018). Perfil epidemiológico de tuberculose em pacientes portadores de HIV. Rev Pre Infec e Saúde, 4, 7519

Almeida, M. R., Barbosa, L. A. O., Casé, M. L. S., \& Pinho, P. S. (2020). Tuberculose Pulmonar no Brasil: uma análise epidemiológica das internações em uma década. Rev. APS, 23 (Supl. 2), 334-335.

Bastos, S. H., Taminato, M., Fernandes, H., Figueiredo, T. M. R. M., Nichiata, L. Y. I, \& Hino, P. (2019). Perfil Sociodemográfico e de saúde da coinfecção tuberculose/HIV no Brasil: revisão sistemática. Rev. Bras. Enferm. 72 (5). https://doi.org/10.1590/0034-7167-2018-0285

Cortez, A. O., Melo, A. C., Neves, L. O., Resende, K. A., \& Camargos, P. (2021). Tuberculose no Brasil: um país, múltiplas realidades. J Bras Pneumol, 47 (2), e20200119.

DATASUS - Tecnologia da Informação a serviço do SUS. http://tabnet.datasus.gov.br/cgi/tabcgi.exe?sinannet/cnv/tubercbr.def.

Ferreira, D. A., Silva, W. B. da, Murilo, B. da C., Andrade Júnior, F. de, Montenegro, C. de A. (2021). Elderly people affected by tuberculosis in the Etate of Paraíba from 2009 to 2019. Research, Society and Development, 10(7), e59210716981. 10.33448/rsd-v10i7.16981. https://rsdjournal.org/index.php/rsd/article/view/16981

Ferreira, T. F., Santos, A. M., Oliveira, B. L. C. A., \& Caldas, A. J. M. (2020). Tendência da tuberculose em indígenas no Brasil no período de $2011-2017$. Ciênc saúde colet 25 (10). https://doi.org/10.1590/1413-812320202510.28482018

Guerra, M. H., Matos, A. C. G., Santos, J. B., Carvalho, D. E., Silva, M. A., Ribeiro, T. M. A., Barreto, C. A. G., Barros, C. A., Barbosa, F. A., \& Jesus, C. V. F. (2021). Covid-19 e tuberculose: coinfecção e riscos. Research, Society and Development, 10 (2), e0710212257, http://dx.doi.org/10.33448/rsdv10i2.122571

Guimarães, A. B. G., Mello, D. C. de, Sousa, L. de, Ângelis C. de, Silva, S. T. F. da, \& Souza, V. de F. (2018). A história da tuberculose associada ao perfil socioeconomico no Brasil: uma revisão da literatura. Caderno de graduação - ciências biológicas e da saúde - UNIT - Pernambuco, 3 (3), 43. https://periodicos.set.edu.br/facipesaude/article/view/5982

Hino, P., Yamamoto, T. T., Bastos, S. H., Beraldo, A. A., Figueiredo, T. M. R. M, \& Bertolozzi, M. R. (2021). Tuberculose na população de rua: revisão sistemática. Rev. esc. enferm. USP, 55. https://doi.org/10.1590/S1980-220X2019039603688

https://agenciabrasil.ebc.com.br. https://agenciabrasil.ebc.com.br/saude/noticia/2020-03/brasil-registra-200-casos-de-tuberculose-por-dia

Lima, S. M. de A., Silva, E. M. de M. e, Lima, M. J. de, \& Jucá A. de M. (2019). Caracterização dos casos de tuberculose notificados em um município prioritário do Brasil, de 2011-2015. Rev Elet Acervo Saúde, 11(13), e482. https://doi.org/10.25248/reas.e482

Maciel, E. L, \& Silva, P. E. A. (2021). Combate à tuberculose: de 1993 a 2035 durante a era da COVID-19. J. bras. pneumol. 47 (02). https://doi.org/10.36416/1806-3756/e20210033

Massabni, A. C., \& Bonini, E. H. (2019). Tuberculose: história e evolução dos tratamentos da doença. Rev bras muldisciplinar, 22(2), 6-34. http://revistarebram.com/index.php/revistauniara

Menezes, R. F., \& Moura, E. R. (2021). Prevalência da tuberculose no Brasil no período de 2011 a 2019. Rev Interdisciplinar em Saúde, 8, 534-545. 10.35621/23587490.v8.n1.p534-545

Moreira, T. R., Lemos, A. C., Colodette, R. M., Gomes, A. P., \& Batista, R. S. (2019). Prevalência de tuberculose na população privada de liberdade: revisão sistemática e metanálise. Rev Panam Salud Publica 43 (10). https://doi.org/10.26633/RPSP.2019.16

Oliveira, C. R., Ribeiro, T. S., \& Dellanhese, A.P.F. (2020). Medidas de prevenção e controle da tuberculose às pessoas privadas de liberdade em presídios brasileiros. Saúde Colet, 10 (58). https://doi.org/10.36489/saudecoletiva.2020v10i58p3817-3830

Santos, M. L., Coeli, C. M., Batista, J. d'A. L., Braga, M. C., \& Albuquerque, M. de F. P. M. de. (2018). Fatores associados à subnotificação de tuberculose com base no Sinan Aids e Sinan Tuberculose. Rev. bras. Epidemiol, 21. https://doi.org/10.1590/1980-549720180019

Silva, D. R., Mello, F. C. de Q., Kritski, A., Dalcolmo, M., Zumla, A., \& Migliori, G. B. (2018). Série Tuberculose. J bras pneumol, 44 (02). https://doi.org/10.1590/S1806-37562018000020001 
Research, Society and Development, v. 10, n. 15, e241101522653, 2021

(CC BY 4.0) | ISSN 2525-3409 | DOI: http://dx.doi.org/10.33448/rsd-v10i15.22653

Silva, L. F., Carvalho, N. S., Souza, B. W. L., Castro, R. C., Feitosa, A. P. A. R., \& Martins, V. H. S. (2020). Fatores de risco e complicações da tuberculose em decorrência do abandono do tratamento. Research, Society and Development, 9 (6), e109963501, http://dx.doi.org/10.33448/rsd-v9i6.3501

Silva, L. T. da, Felipini, M. C. C., Oliveira, T. B. de, Brunello, M. E. F., \& Orfão, N. H. (2019). Perfil epidemiológico da tuberculose no serviço de referência do estado de Rondônia. Rev. epidemiol. controle infecç, 9 (1), 48-54. https://pesquisa.bvsalud.org/portal/resource/pt/biblio-1021166

Silva, P. H. dos S, Cirilo, S. S. V., Sousa Júnior, S. C. de, Cruz, V. T., Correia, R. S., Santos, A. F. dos, Costa, T. R. M., Vieira, C. G., \& Miranda, A. S. (2020). Aspectos sociodemográficos e clínicoepidemiológicos da tuberculose em um município do nordeste brasileiro. Rev Elet Acervo Saúde, $12(5)$, e1916. https://doi.org/10.25248/reas.e1916.2020

SINAN - Sistema de Informação de Agravo de Notificação. http://portalsinan.saude.gov.br/tuberculose.

Sousa, F. das C. A., Soares, H. V. A., Lemos, L. E. A. S., Reis, D. M., Silva, W. C. da, \& Rodrigues, L. A. de S. (2020). Epidemiological profile of neglected mandatory reporting diseases in Brazil with analysis of government investments in this área. Research, Society and Development, 9(1), e62911610. https://doi.org/10.33448/rsd-v9i1.1610

World Health Organization [homepage on the Internet]. Geneva: World Health Organization, c2017. Global tuberculosis report 2017. http://www.who.int/tb/publications/global_report/gtbr2017_main_text.pdf 